\title{
EpiLog: A software for the logical modelling of epithelial
}

\section{dynamics [version 1; peer review: 2 approved, 1 approved with}

\section{reservations]}

\author{
Pedro L. Varela1-3, Camila V. Ramos2,3, Pedro T. Monteiro (D1,2, \\ Claudine Chaouiya (1D 3 \\ ${ }^{1}$ Instituto Superior Técnico, Universidade de Lisboa, Lisbon, P-1049-001, Portugal \\ 2INESC-ID, Lisbon, P-1000-029, Portugal \\ 3Instituto Gulbenkian de Ciência, Oeiras, P-2780-156, Portugal
}

\author{
V1 First published: 27 Jul 2018, 7:1145 \\ https://doi.org/10.12688/f1000research.15613.1 \\ Latest published: 11 Mar 2019, 7:1145 \\ https://doi.org/10.12688/f1000research.15613.2
}

Open Peer Review

Approval Status

1

2

3

version 2

(revision)

11 Mar 2019

version 1

27 Jul 2018

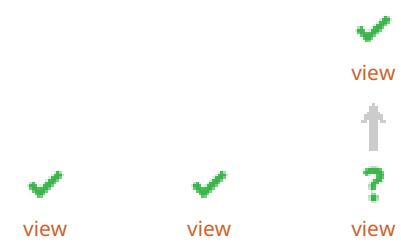

1. Stefano Nichele, Oslo Metropolitan

University, Oslo, Norway

2. Alejandro F. Villaverde (ID), IIM-CSIC, Vigo, Spain

David Henriques (D), IIM-CSIC, Vigo, Spain

3. Arnau Montagud ID, PSL Research

University, Paris, France

Barcelona Supercomputing Center (BSC),

Barcelona, Spain

Any reports and responses or comments on the article can be found at the end of the article. 
Corresponding authors: Pedro T. Monteiro (Pedro.Tiago.Monteiro@tecnico.ulisboa.pt), Claudine Chaouiya (chaouiya@igc.gulbenkian.pt )

Author roles: Varela PL: Software, Writing - Original Draft Preparation, Writing - Review \& Editing; Ramos CV: Software, Writing Review \& Editing; Monteiro PT: Funding Acquisition, Software, Supervision, Writing - Original Draft Preparation, Writing - Review \& Editing; Chaouiya C: Conceptualization, Funding Acquisition, Supervision, Writing - Original Draft Preparation, Writing - Review \& Editing

Competing interests: No competing interests were disclosed.

Grant information: This work was supported by national funds through Fundação para Ciência e a Tecnologia (FCT) with reference PTDC/BEX-BCB/0772/2014, UID/CEC/50021/2013 and IF/01333/2013. PV was supported by grant PTDC/BEX-BCB/0772/2014 and PTDC/EIA-CCO/099229/2008. CR was supported by PTDC/BEX-BCB/0772/2014 and IF/01333/2013/CP1204/CT0001. Furthermore, CC and CR acknowledge support from the Fundação Calouste Gulbenkian.

The funders had no role in study design, data collection and analysis, decision to publish, or preparation of the manuscript.

Copyright: ( 2018 Varela PL et al. This is an open access article distributed under the terms of the Creative Commons Attribution License, which permits unrestricted use, distribution, and reproduction in any medium, provided the original work is properly cited.

How to cite this article: Varela PL, Ramos CV, Monteiro PT and Chaouiya C. EpiLog: A software for the logical modelling of epithelial dynamics [version 1; peer review: 2 approved, 1 approved with reservations] F1000Research 2018, 7:1145 https://doi.org/10.12688/f1000research.15613.1

First published: 27 Jul 2018, 7:1145 https://doi.org/10.12688/f1000research.15613.1 


\section{Introduction}

Pattern formation emerges from the interplay of interaction networks, at the cellular and multi-cellular levels ${ }^{1}$. To uncover the complex mechanisms at stake, computational modelling is very needed. In this context, cellular automaton approaches are particularly well suited ${ }^{2}$.

On the other hand, the logical formalism has proved efficient to explore cellular regulatory networks driving developmental processes (for a recent review on the logical modelling approach, see 3). The consideration of ensembles of communicating cells is often required to recapitulate observed patterns (e.g. 4,5).

This motivated the development of EpiLog, which implements an extension of the logical framework to hexagonal grids embodying simple epithelia.

Briefly, a logical model assigns discrete (Boolean or multivalued) variables to the regulatory components, and logical regulatory rules define the evolution of the variables depending on the variables associated with the component regulators. Input components embody cell receptors receiving external signals. Unlike internal components, inputs have no associated regulatory rules, and are generally maintained at constant values accounting for fixed environmental conditions. A model state is given by the values of the components, and the dynamics is generated according to specific updating schemes. In a synchronous update, a state has at most one successor state in which all the variables have been updated as prescribed by the regulatory rules. In contrast, the asynchronous update generates as many successors as the number of updated variables, yielding more complex and non-deterministic behaviours. Properties of the resulting dynamics relate to the model attractors (stable states or point attractors, and cyclical attractors), which can be associated with different phenotypes. Beyond identifying the attractors, a major question relates to reachability properties: given an initial condition, what is (are) the reachable attractor(s)?

The definition, analysis and simulation of logical models of cellular networks can be performed using one of the existing software tools ${ }^{6,7}$. Many of these support the exchange format SBML qual (Qualitative Models package for SBML) $)^{7,8}$.

To handle multi-cellular systems, Mendes et al. have established a compositional approach relying on a process algebra framework to assess stable states reachability in asynchronous dynamics ${ }^{9}$. This approach showed some limitations due to the huge size of the considered state spaces, and is thus limited to the analysis of models encompassing a reduced number of cells (up to a dozen cells). Nonetheless, in the cellular automaton framework considered here, we use the same approach to specify cell-cell communication through (logical) integration rules. These govern the evolution of cellular input components, depending on the values of internal components (representing e.g. secreted proteins) in neighbouring cells.

\section{Methods}

\section{Implementation}

EpiLog is implemented in Java (requires Java 7 or higher), is freely available at http://epilog-tool.org, and launches a Graphical
User Interface (GUI) based on JFC/Swing. It is provided as a single.jar file which can be launched from a graphical file manager by double clicking on it or through the command line as follows:

\$ java -jar EpiLog-versionx.jar [--file /path/to/ multicellular_project.peps]

where the flag --file can be used to optionally specify the file to be open. EpiLog .peps files represent projects containing multiple multicellular models, each with potentially distinct characteristics.

Additionally, an EpiLog model repository is provided at http:// epilog-tool.org/models_repository where users can deposit their models by contacting support@epilog-tool.org. Each model page includes a title, a description, a taxon and process classification, model . peps file(s) and supporting paper(s).

Developers can freely access the code repository at http:// github.com/epilog-tool/epilog, clone it and extend the code. For dependency management of external libraries, EpiLog relies on Apache Maven. A particular library is the bioLQM Java toolkit (Logical Qualitative Models of biological regulatory networks), for the representation and manipulation of logical, cellular models, available at https://github.com/colomoto/bioLQM ${ }^{10}$.

\section{Operation}

Model definition EpiLog defines projects that include models, called epithelia, each being specified by:

1. The size of the hexagonal grid, as well as its border conditions; vertical and horizontal borders can be connected, or not leading to rectangular, cylindrical or toroidal grids;

2. A (set of) logical model(s), each associated with cells of the grid; these cellular models can be defined in any tool supporting the SBML qual format ${ }^{8}$. EpiLog imports SBML files and identifies input components in these cellular models;

3. The cellular input components; these are defined as constant positional inputs (e.g. morphogens produced by external sources) or evolving integration inputs; for the latter, logical integration rules specify which signals are received from (internal) components of neighbouring cells and how these signals are combined;

4. An initial state defining the values of the internal components of all the cells.

Simulation settings For each epithelium, simulation settings define the updating schemes for both the cellular and epithelium models. The default scheme is synchronous, that is to say, at each iteration, all the cellular models are considered for a synchronous update of their internal components. To overcome spurious oscillations generated by these synchronous updates (see Figure 1A and Supplementary File 1), EpiLog allows to specify:

1. The cellular model update, with the definition of (synchronous) priority classes (increasing or decreasing) 


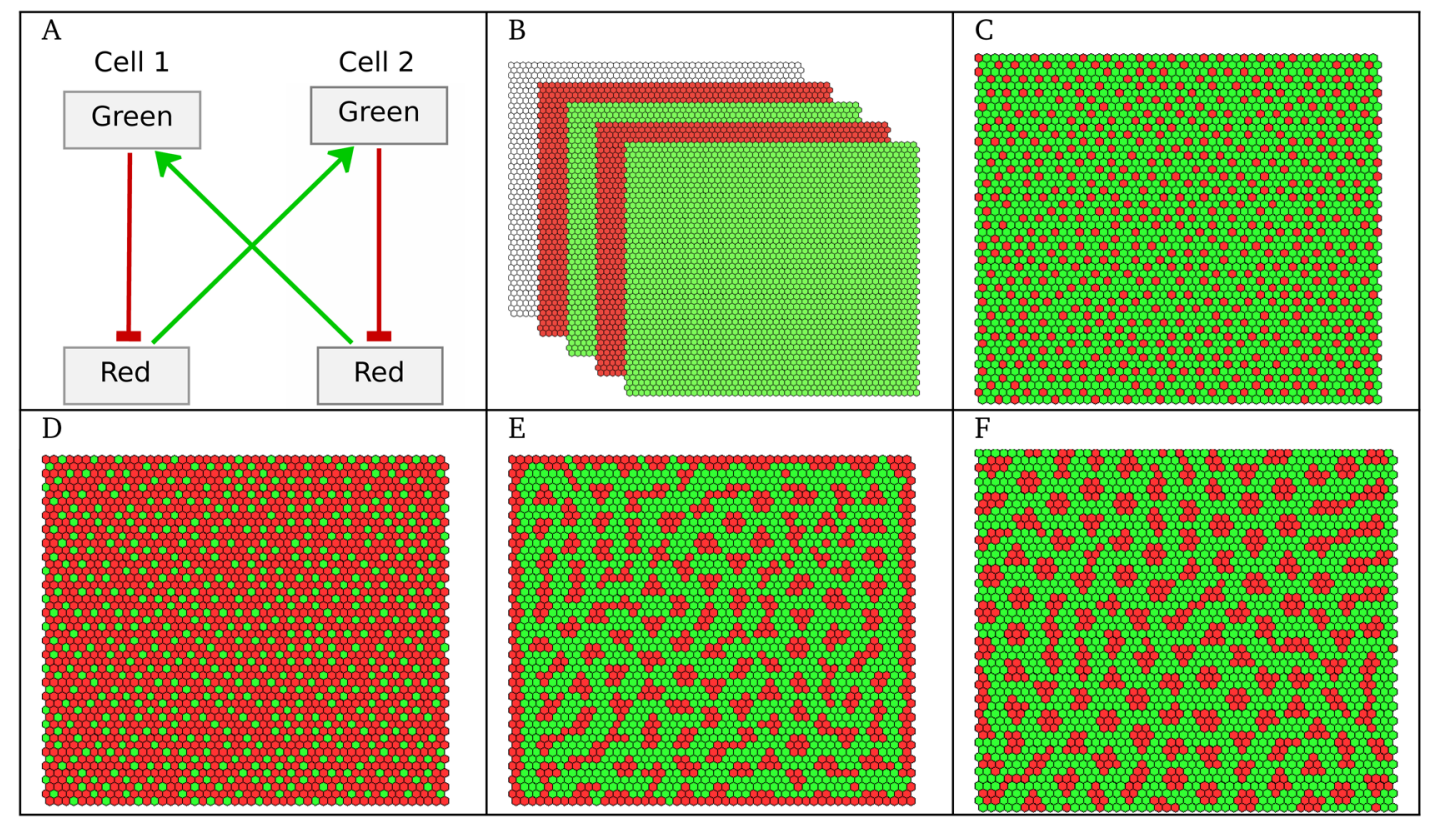

Figure 1. (A) An idealised Boolean model of lateral inhibition; the Green marker is induced by the Red marker secreted by the neighbouring cell, whereas Red is induced by low levels of Green in the same cell; the synchronous dynamics of this two cell model leads to a cyclic attractor (see Supplementary File 1). (B) Simulation in EpiLog, with the same cellular model over a square grid of $50 \times 50$ hexagonal cells under a synchronous update (Green induced if at least one contacting cell is Red), oscillations are due to the synchronous update of the cells, see Supplementary Movie 1. (C-F) Simulations under $\alpha$-asynchrony $(\alpha=0.25)$ : (C) Stable pattern reached in 29 steps (Green induced if at least one contacting cell is Red), see Supplementary Movie 2; (D) Stable reverted pattern reached in 30 steps (Green induced if all contacting cells are Red); (E) Stable pattern reached in 63 steps (Green induced if at least 12 cells at distance up to 3 are Red); (F) Stable pattern reached in 70 steps, with the same setting as for panel E but considering a torus (i.e., no grid borders).

updates are gathered in ordered sets, allowing to account for different time scales for the mechanisms underlying component updates ${ }^{11}$;

2. The epithelium update, with the definition of probabilistic updates of the cell states, inspired by N. Fatès' alpha-asynchronism ${ }^{12}$ : parameter $\alpha$ specifies the proportion of the cells randomly chosen for update.

Model perturbations The logical formalism permits to easily specify perturbations (e.g. knock-out or ectopic expression of cellular model components) by blocking the values of perturbed components. EpiLog includes this feature with the definition of component perturbations that can be applied to all the cells or to restricted regions of the grid.

The reader is invited to consult the documentation available at EpiLog web site to get further information (http://epilog-tool. org/documentation).

\section{Use cases}

As a first illustration, we consider a very simple model, in which the cellular model includes two markers. When considering a juxtacrine signal (i.e., only contacting cells can communicate), this model accounts for the well-known Delta-Notch signalling, which implements a lateral inhibition process (see e.g. 13). The model is provided in Supplementary Model
File 1. In Figure 1A, a two-cell model is shown, to illustrate interactions within and between the cells. Remaining panels of Figure 1 show EpiLog simulation results, illustrating the different patterns obtained when varying the updating mode, the integration rules and the border conditions.

The development of EpiLog was originally motivated by our second illustration, which relates to the dorsal appendageforming regions of the Drosophila Melanogaster eggshell ${ }^{14}$ (see Figure 2). The logical model defined by Fauré et al. reproduces the patterning of the anterior follicular epithelium of the oocyte, defining the floor (Rho expressing cells) and roof (Br expressing cells) of the future appendages ${ }^{5}$. The model is provided in Supplementary Model File 2. EpiLog window is shown in Figure 2A, with the simulation panel displaying the stable pattern obtained with this model. The remaining panels C-G show different states of the grid, with panels $\mathrm{F}-\mathrm{G}$ recapitulating patterns resulting from model perturbations (see Figures 6-8 in 5).

\section{Summary}

To the best of our knowledge, EpiLog is the first software tool for the definition, simulation and visualisation of qualitative, logical (Boolean and multivalued) models over hexagonal grids. It provides a graphical user interface and tools to conveniently support the study of epithelial pattern formation, relying on a logical framework. 


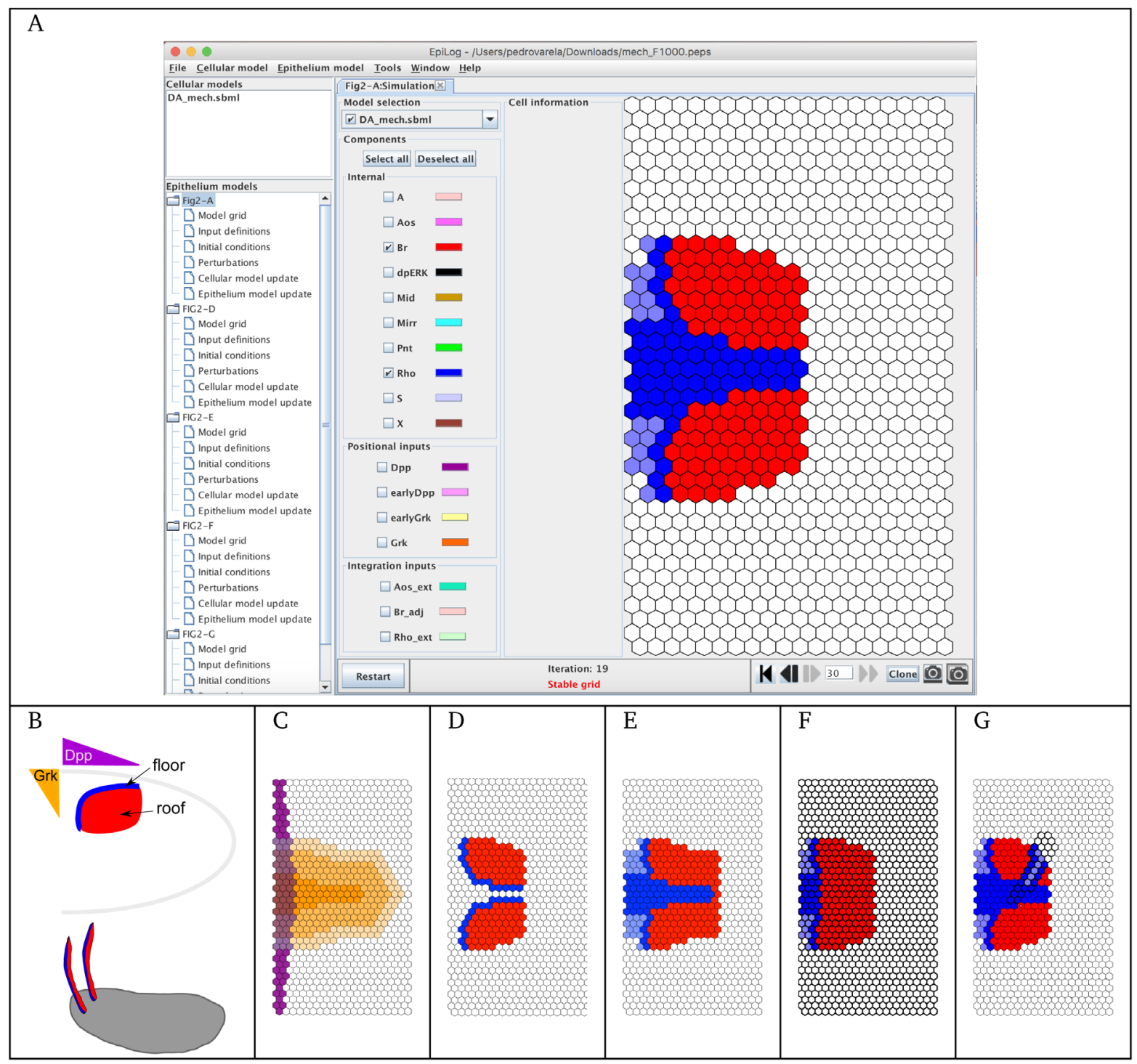

Figure 2. Model of the fly eggshell patterning of Fauré et al. ${ }^{5}$, implemented in EpiLog. (A) EpiLog main window with the stable pattern reached by a simulation starting with all the cells of the grid having their internal components at 0 , and positional inputs defined as shown in panel C (phase 1). (B) Egg chamber with two dorsal respiratory appendages (DA); DA primordia are established as 2 regions on both sides of the oocyte midline, with follicle cells expressing Broad (Br in red), future roof of the DA, and cells expressing Rhomboid (Rho in blue), future floor of the DA. Establishment of these regions involve Gurken signalling (Grk, in orange) and Decapentaplegic signalling (Dpp, in violet). (C) Grk and Dpp gradient defined as positional inputs in EpiLog. (D) Stable pattern obtained with a simulation starting from the pattern displayed in panel A and without the Grk signal (phase 2), suggesting that Grk extinction is required to split the floor regions (see 5). (E) EpiLog simulation of a mild overexpression of Dpp. (F) EpiLog simulation of Pointed (Pnt, internal component) loss-of-function. (G) EpiLog simulation of Pnt gain-of-function clones. Note that in panels $\mathbf{F}-\mathbf{G}$, perturbed cellular models are indicated by bold borders in the grid.

EpiLog is free, open source and implemented in Java for operating system independence. It relies on the SBML qual standard format to import cellular models.

\section{Software availability}

EpiLog v1.1 is available from: http://epilog-tool.org/downloads

Source code available from: https://github.com/epilog-tool/epilog
Archived source code as at time of publication: https://doi. org/10.5281/zenodo. $1320503^{15}$

License: GNU General Public License v3.0

\section{Author information}

PV and CR developed the software. PTM acquired funding, developed the software and supervised the project. CC designed the 
project, acquired funding and supervised the project. PLV, CVR, PTM and CC wrote the article.

\section{Competing interests}

No competing interests were disclosed.

\section{Grant information}

This work was supported by national funds through Fundação para Ciência e a Tecnologia (FCT) with reference PTDC/BEXBCB/0772/2014, UID/CEC/50021/2013 and IF/01333/2013. PLV was supported by grant PTDC/BEX-BCB/0772/2014 and PTDC/EIA-CCO/099229/2008. CVR was supported by PTDC/
BEX-BCB/0772/2014 and IF/01333/2013/CP1204/CT0001. Furthermore, CC and CVR acknowledge support from the Fundação Calouste Gulbenkian.

The funders had no role in study design, data collection and analysis, decision to publish, or preparation of the manuscript.

\section{Acknowledgments}

The authors would like to thank Adrien Fauré who implemented a first Python prototype that inspired the development of EpiLog, and who provided insightful feedback. We are also grateful to Céline Hernandez for her useful comments.

\section{Supplementary material}

Supplementary File 1: Dynamics of the lateral inhibition model under synchronous and asynchronous updates.

Click here to access the data.

Supplementary Movie 1: Oscillatory behaviour of the lateral inhibition model under a synchronous update (see Figure 1 panel B).

Click here to access the data.

Supplementary Movie 2: Stable pattern of the lateral inhibition model under an $\alpha$-asynchronous update (see Figure 1 panel C).

Click here to access the data.

Supplementary Model File 1: EpiLog file including variations of the lateral inhibition model as illustrated in Figure 1.

Click here to access the data.

Supplementary Model File 2: EpiLog file including variations of the DA model as illustrated in Figure 2.

Click here to access the data.

1. Kicheva A, Cohen M, Briscoe J: Developmental pattern formation: insights from physics and biology. Science. 2012; 338(6104): 210-2.

PubMed Abstract | Publisher Full Text

2. Deutsch A, Dormann S: Cellular automaton modeling of biological pattern formation: characterization, applications, and analysis. Birkhäuser Boston, 2005.

Publisher Full Text

3. Abou-Jaoudé W, Traynard P, Monteiro PT, et al:: Logical Modeling and Dynamical Analysis of Cellular Networks. Front Genet. 2016; 7: 94. PubMed Abstract | Publisher Full Text | Free Full Text

4. Sánchez L, Chaouiya C, Thieffry D: Segmenting the fly embryo: logical analysis of the role of the segment polarity cross-regulatory module. Int J Dev Biol. 2008; 52(8): 1059-75.

PubMed Abstract | Publisher Full Text

5. Fauré A, Vreede BM, Sucena E, et al:: A discrete model of Drosophila eggshell patterning reveals cell-autonomous and juxtacrine effects. PLOS Comput Biol. 2014; 10(3): e1003527.

PubMed Abstract | Publisher Full Text | Free Full Text

6. Naldi A, Monteiro PT, Müssel C et al: Cooperative development of logical modelling standards and tools with CoLoMoTo. Bioinformatics. 2015; 31(7): 1154-9. PubMed Abstract | Publisher Full Text
7. Chaouiya C, Bérenguier D, Keating SM, et al: SBML qualitative models: a model representation format and infrastructure to foster interactions between qualitative modelling formalisms and tools. BMC Syst Biol. 2013; 7: 135. PubMed Abstract | Publisher Full Text | Free Full Text

8. Chaouiya C, Keating SM, Berenguier D, et al.: The Systems Biology Markup Language (SBML) Level 3 Package: Qualitative Models, Version 1, Release 1. $J$ Integr Bioinform. 2015; 12(2): 270.

PubMed Abstract | Publisher Full Text

9. Mendes ND, Lang F, Le Cornec YS, et al.: Composition and abstraction of logical regulatory modules: application to multicellular systems. Bioinformatics. 2013; 29(6): 749-57.

PubMed Abstract | Publisher Full Text

10. Naldi A: bioLQM: a java library for the manipulation and conversion of Logical Qualitative Models of biological networks. bioRxiv. 2018; 287011. Publisher Full Text

11. Fauré A, Naldi A, Chaouiya C, et al: Dynamical analysis of a generic Boolean model for the control of the mammalian cell cycle. Bioinformatics. 2006; 22(14): e124-131. PubMed Abstract | Publisher Full Text

12. Fatès N: A Guided Tour of Asynchronous Cellular Automata. In Cellular Automata and Discrete Complex Systems - 19th International Workshop, AUTOMATA 2013, Gießen, Germany, September 17-19, 2013. Proceedings. 2013; 
$15-30$.

Publisher Full Text

13. Collier JR, Monk NA, Maini PK, et al.: Pattern formation by lateral inhibition with feedback: a mathematical model of delta-notch intercellular signalling. $J$ Theor Biol. 1996; 183(4): 429-46.

PubMed Abstract | Publisher Full Text
14. Pyrowolakis G, Veikkolainen V, Yakoby N, et al:: Gene regulation during Drosophila eggshell patterning. Proc Natl Acad Sci U S A. 2017; 114(23): 5808-5813. PubMed Abstract | Publisher Full Text | Free Full Text

15. Varela PL, Monteiro PT, Ramos CV: epilog-tool/epilog: EpiLog v1.1 (Version v1.1). Zenodo. 2018

http://www.doi.org/10.5281/zenodo.1320503 


\title{
Open Peer Review
}

\section{Current Peer Review Status:}

\section{Version 1}

Reviewer Report 10 October 2018

https://doi.org/10.5256/f1000research.17032.r37601

(c) 2018 Montagud A. This is an open access peer review report distributed under the terms of the Creative Commons Attribution License, which permits unrestricted use, distribution, and reproduction in any medium, provided the original work is properly cited.

\author{
Arnau Montagud \\ ${ }^{1}$ Institut Curie, Inserm U900, Mines Paris Tech, PSL Research University, Paris, France \\ 2 Barcelona Supercomputing Center (BSC), Barcelona, Spain
}

The paper describes a software used for qualitative simulations of logical models in a hexagonal grid, called EpiLog. The paper is well written, even though some parts could be slightly expanded for a better comprehension. The software is easy to use for people with logical modelling background and is a visual way of exploring populations of Boolean models and their effect on neighbouring cells. The software is freely available, cross-platform, can use open standards of logical models and its code can be studied and forked in GitHub.

Nevertheless, the paper would benefit from some additions and clarifications:

Major:

1. Expansion of introduction: The last sentence of first paragraph could be completed with some of the reasons cellular automaton are well suited. Also, the relationship of present paper and [1] could be better worded. It is unclear if authors used Mendes et al approach as is or if they improved Mendes et al approach and can work with more than a dozen cells. Addressing these would enhance the understanding of the work.

2. Discussion on the alpha parameter: The paper would benefit from a discussion on the use of "alpha-asynchronism" as logical modelling tools, until now, either used asynchronous and synchronous updates. Also, the paper presents results using alpha=1 (pure synchronous) or 0.25 , but does not present results with alpha $=0$ (pure asynchronous). Presenting and discussing these (even in Supplementary) would allow users to have a better understanding on the effects of this parameter.

3. Detailed tutorial: Even though the Documentation does a good job detailing the possibilities of the tool, the software users would benefit from a detailed discussion on the effect of changing spatial initial conditions, alpha parameter and, in general, the biological relevance of these. This supplementary file would also allow to expand on the discussion of Figure 2 

Minor:

that is absent from the paper (understandably being a software tool paper).

1. Comparison to other software: Even though this software is the first that includes hexagonal grid and Boolean models, naming some other software that can be used for similar projects (with their pros and cons) would give the reader a better sense on the current status of the field.

2. In Methods/Operation/Simulation settings, first sentence, authors say "For each epithelium, simulation settings define the updating schemes for both the cellular and epithelium models." Does cellular model mean one cell and epithelium model the whole set of cells? As the phrase starts with epithelium, the current wording is a bit confusing.

3. Figure 1 legend: "Stable pattern" is a bit confusing, maybe it would be better to use "pattern of stable states".

4. Figure 2 legend: Authors should describe "phase 1" and "phase 2 " in the paper to understand what is the difference in panels $C$ and $D$. In panel $B$, "DA" should be shown in the figure. In panel C, the colours for Grk and Dpp should be explained (even if they are described in previous panel).

5. In Supplementary text, in the last part of the last sentence, the Figure in main text you refer to is Figure 2, panels C-F.

Following previous reviewers' comments, I had no errors using EpiLog on Windows.

\section{References}

1. Mendes ND, Lang F, Le Cornec YS, Mateescu R, et al.: Composition and abstraction of logical regulatory modules: application to multicellular systems.Bioinformatics. 2013; 29 (6): 749-57

PubMed Abstract | Publisher Full Text

Is the rationale for developing the new software tool clearly explained?

Yes

Is the description of the software tool technically sound?

Yes

Are sufficient details of the code, methods and analysis (if applicable) provided to allow replication of the software development and its use by others?

Yes

Is sufficient information provided to allow interpretation of the expected output datasets and any results generated using the tool?

Partly

Are the conclusions about the tool and its performance adequately supported by the findings presented in the article? 
Yes

Competing Interests: No competing interests were disclosed.

Reviewer Expertise: Systems Biology, Cancer, Boolean modelling, Multiscale modelling, Metabolic modelling, Data deconvolution

I confirm that I have read this submission and believe that I have an appropriate level of expertise to confirm that it is of an acceptable scientific standard, however I have significant reservations, as outlined above.

Author Response 28 Feb 2019

Claudine Chaouiya, Instituto Gulbenkian de Ciência, Oeiras, Portugal

We thank you for the detailed comments and suggestions. We have revised the manuscript accordingly, and we briefly answer your points below.

We have revised the introduction, noting that cellular automata are a natural modelling choice to define local rules governing the behaviours of cells over a lattice. We also clarified the link between the framework as implemented in EpiLog and the work presented in Mendes et al 2013.

We have included a supplementary text illustrating and discussing the use of the alphaasynchronous update, and varying the values of alpha. This is done using the simple model of lateral inhibition. It is worth to note that a general statement of how the value of alpha impacts the dynamics (intermediate and final patterns) is hard to make, as such properties certainly depend on the model. Finally, alpha-asynchronism has been considered for cellular automata. The reviewer is right when noting that this update has not been employed for (cellular) logical models but, besides a theoretical interest, it is unclear if such an update would have any biological significance besides the fact that indeed a few cells may update their states simultaneously.

Concerning your point on a detailed tutorial, in addition to the documentation, we now provide some video to illustrate the major steps in defining and simulating a model with EpiLog (available at https://www.youtube. com/channel/UCGGcpepqyYJkt7dhrhncQiQ, as indicated in the documentation). Results displayed in Figure 2 are thoroughly discussed in the original paper, Fauré et al. 2014.

We now mention the two other software tools we are aware of that consider logical models in a multi-scale setting.

We have clarified the terminology. In particular we have changed the legend of Fig 1, which now says "stable state of the grid, referred to as stable pattern". Indeed a stable pattern is defined by stable states of the cells, but not any combination of such cellular stable states remain stable in the multi-cellular model.

Concerning Fig. 2, the requirement for the 2 phases is now shortly described in the main 
text. The legend has been modified as suggested, and dorsal appendage (DA) has been added in panel $\mathrm{B}$.

Competing Interests: No competing interests were disclosed.

Reviewer Report 04 October 2018

https://doi.org/10.5256/f1000research.17032.r38246

(C) 2018 Villaverde A et al. This is an open access peer review report distributed under the terms of the Creative Commons Attribution License, which permits unrestricted use, distribution, and reproduction in any medium, provided the original work is properly cited.

\section{Alejandro F. Villaverde}

Bioprocess Engineering Group, IIM-CSIC, Vigo, Spain

\section{David Henriques}

Bioprocess Engineering Group, IIM-CSIC, Vigo, Spain

This paper describes a new software tool for modelling epithelial dynamics in 2D. The software, called EpiLog, uses a logical framework that assigns discrete values to the model variables. The approach used for specifying the logical rules was previously presented in a paper from the same senior author (Mendes et $\mathrm{al}^{1}$ ).

The paper is well written, and the software is provided in a nice dedicated website, which also includes documentation and additional information. Furthermore, the source code is provided at GitHub.

We have a few comments and suggestions regarding both the methodological approach and the software implementation:

In regard to the method:

1) It is stated that the published method can only handle up to a dozen cells. However, the graphical interface seems to describe more. Maybe the authors could elaborate on the limitations of the tool, as well as on its performance for different types of problems.

2) It is not totally clear whether the approach used in this paper is exactly the same as the one published in (Mendes et $\mathrm{al}^{1}$ ). If it is not, and if there is no other published paper describing the method, an upgraded methods paper could be of interest. On the other hand, if there is another publication describing the method, it should be cited in the paper. It is also unclear if the method has been previously validated in epithelium (hexagonal) case. The authors may want to clarify these points in a revised version. 
3) We are aware that, being a software tool article, the present paper is probably not the best place for extended discussions about biological aspects. The following points may therefore be considered as suggestions for the content of an upgraded paper as described in the previous paragraph. Of course, they would also be welcome additions to an extended version of the present paper, if the authors deem them appropriate to address:

3.1) It may be useful to describe how to define a new (toy) model and a grid from scratch.

3.2) What type of cues/signals are considered?

3.3) A real case study would make it easier to follow the pipeline.

3.4) Some additional biological explanation or motivation of potential applications might make the paper more attractive.

In regard to the software:

1) We tested EpiLog in Windows. We managed to load and simulate the Drosophila "Eggshell Patterning", but the software reported warnings/errors. Are these relevant?

\section{References}

1. Mendes ND, Lang F, Le Cornec YS, Mateescu R, et al.: Composition and abstraction of logical regulatory modules: application to multicellular systems.Bioinformatics. 2013; 29 (6): 749-57

PubMed Abstract | Publisher Full Text

Is the rationale for developing the new software tool clearly explained?

Yes

Is the description of the software tool technically sound?

Yes

Are sufficient details of the code, methods and analysis (if applicable) provided to allow replication of the software development and its use by others?

Yes

Is sufficient information provided to allow interpretation of the expected output datasets and any results generated using the tool?

Partly

Are the conclusions about the tool and its performance adequately supported by the findings presented in the article?

Yes

Competing Interests: No competing interests were disclosed.

Reviewer Expertise: David Henriques: dynamic modelling, bioinformatics, logic-based modelling, 
systems biology. Alejandro F Villaverde: dynamic modelling, system identification, systems biology, control theory.

\section{We confirm that we have read this submission and believe that we have an appropriate level of expertise to confirm that it is of an acceptable scientific standard.}

\section{Author Response 28 Feb 2019}

Claudine Chaouiya, Instituto Gulbenkian de Ciência, Oeiras, Portugal

We thank you for the comments and suggestions, and we briefly answer each point or the report below.

1) We believe the misunderstanding was due to the confusion with our mention to a previous work (Mendes at al 2013). This should now be clarified.

2) This should be clear in the revised text. The rationale to define cell-cell communication (i.e. the rules governing the behaviours of input components of cellular models) relies on the definitions presented in Mendes et al 2013. However, EpiLog provides a graphical 1 interface and a simulation platform, producing a single model trajectory, whereas Mendes et al. aimed at determining all the stable states reachable from a given initial condition and considering an asynchronous update.

3) 3.1 should now be addressed through the video that are available on EpiLog web site 3.2 integration inputs are now more explicit in the text (juxtacrine signals, or other secreted molecules influencing cells in the neighbourhood)

3.3 the DA formation is a real case study. The interested reader can refer to the original paper by Fauré et al., and can also download the model from EpiLog web site.

3.4 Indeed, we intend to include further arguments on biological applications in a forthcoming paper on lattice-based modelling approaches

4) Regarding the software: EpiLog relies on bioLQM, which in turn relies on JSBML libraries. The warning messages are being thrown by JSBML when loading a cellular model, but do not affect any of EpiLog's behaviour.

Competing Interests: No competing interests were disclosed.

Reviewer Report 20 August 2018

https://doi.org/10.5256/f1000research.17032.r37297

(C) 2018 Nichele S. This is an open access peer review report distributed under the terms of the Creative Commons Attribution License, which permits unrestricted use, distribution, and reproduction in any medium, provided the original work is properly cited. 


\section{Stefano Nichele}

Department of Computer Science, Oslo Metropolitan University, Oslo, Norway

The article describes EpiLog, a software tool to model artificial development of epitelial tissue through cellular automata with hexagonal cells.

EpiLog provides a high degree of customization through the graphical user interface. This includes: (a) model definition, such as CA size, logical models and initial states; (b) simulation settings, including updates (synchronous or asynchronous/probabilistic) and (c) perturbations on single cells or regions.

The article provides two simple use cases to introduce the user to the software. First, a model of lateral inhibition process is presented in FIg. 1. Second, a developmental model of Drosophila eggshell.

\section{Minor revisions:}

The article would benefit by the following:

background section with related work and description of CA/artificial development software tools, with brief description of similarities and differences;

a more advanced use case described in details (for the advanced reader).

Is the rationale for developing the new software tool clearly explained?

Yes

Is the description of the software tool technically sound?

Yes

Are sufficient details of the code, methods and analysis (if applicable) provided to allow replication of the software development and its use by others?

Yes

Is sufficient information provided to allow interpretation of the expected output datasets and any results generated using the tool?

Yes

Are the conclusions about the tool and its performance adequately supported by the findings presented in the article?

Yes

Competing Interests: No competing interests were disclosed.

Reviewer Expertise: Artificial Life, Complex Systems, Evo-Devo, Cellular Automata

I confirm that I have read this submission and believe that I have an appropriate level of 
expertise to confirm that it is of an acceptable scientific standard.

Author Response 28 Feb 2019

Claudine Chaouiya, Instituto Gulbenkian de Ciência, Oeiras, Portugal

We than you for the suggestions. We considered that a proper state of the art was beyond the scope of this application note. However, we now acknowledge the existence of two closely related tools, CoGNaC and PhysiBoSS. The DA formation is a real, advanced case study. The interested reader can refer to the original paper by Fauré et al., and can also download the model from EpiLog web site.

Competing Interests: No competing interests were disclosed.

The benefits of publishing with F1000Research:

- Your article is published within days, with no editorial bias

- You can publish traditional articles, null/negative results, case reports, data notes and more

- The peer review process is transparent and collaborative

- Your article is indexed in PubMed after passing peer review

- Dedicated customer support at every stage

For pre-submission enquiries, contact research@f1000.com 